\title{
The vertical-horizontal illusion in a visually-rich environment
}

ALPHONSE CHAPANIS AND DONALD A. MANKIN

THE JOHNS HOPKINS UNIVERSITY

In the vertical-horizontal illusion the vertical dimension of a figure typically appears longer than the horizontal. Although there is a large body of research literature on this illusion, all of it refers to simple figures with well-drawn lines, exhibited against plain backgrounds. Our experiment has investigated the illusion using real-world objects in a visually-rich environment. Ten male and 10 female $S$ s were asked to judge the heights of 10 objects of various sizes and shapes. They made their estimates by having the experimenter mark off a horizontaldistance that corresponded to the judged height of the object. Each $S$ estimated the height of each object once a day for three consecutive days. The results show that, by and large, the illusion can be demonstrated for real objects in a visually-rich environment. There was, however, considerable variation among the objects. It appears that estimates of this kind may be influenced by size, size constancy, anchor effects, and angle of regard, along with other, as yet, unidentified factors.

The vertical-horizontal illusion is widely cited as one of the ways in which we misperceive the world around us. A simple form of the lllusion consists of an inverted- $T$ figure in which the vertical and horizontal lines are of equal length (Fig. 1, left). Most Ss judge the vertical line to be longer than the horizontal one. The illusion has also been demonstrated in L-shaped figures (Fig. 1, right) although the vertical overestimation in this case is not quite as prominent as in the inverted-T figure.

In the more than one hundred years since Fick (see Künnapas, 1957) first drew attention to it, many different attempts have been made to explain this illusion and its various forms. One of the more convincing theories is that of Künnapas (1957), who explains the illusion by attributing it to the shape of our visual field. Because the shape of the visual field is oval, the ends of the vertical line of the inverted-T or L-shaped figures lie closer to the boundary of the visual field than do the edges of the horizontal line. Künnapas says that it is the relative "nearness" of the vertical line to the boundary of the field that causes its length to be overestimated when compared with the length of the horizontal line.

In the large amount of experimental literature on this illusion we have found no studies that have tried to relate it to the perception of real objects in the everyday environment. Typically, Es have used simple figures with well drawn lines (like those in Fig. 1), and have exhibited these figures against plain back- grounds. No one has asked the practical question, "Can the illusion be demonstrated with real objects in a visually-rich environment?"

There have been some suggestions that the overestimation of vertical lines is influenced by more than just the shape of the visual field. Pan (1934), for example, was one of the first to emphasize that the illusion is, in part, due to the peculiar characteristics of the flgure itself. The size of the test figure also appears to be an important variable. With figures ranging from 8 to $200 \mathrm{~mm}$ in size (but with viewing distance. unspecified) Pan concluded that the larger the figure the less the illusion. Finger and Spelt (1947) and Künnapas (1955) found that in the case of the inverted-T figure an additional influence is exerted by the bisection of the horizontal line by the vertical one. Finally there is the study by Sleight and Austin (1952) who investigated the illusion with two-dimensional geometric figures. They found that the vertical dimensions of circles and ellipses-but not of squares and rectangles-were overestimated when compared with their horizontal dimensions.

Studies such as those cited above suggest that many factors may affect this illusion. It seems reasonable to assume that more of these factors would be present in the visually-rich, everyday environment than in the abstract setting of the laboratory. Starting with these premises, then, the purpose of the present experiment was to determine whether we can generalize from the lllusion demonstrated in the laboratory to the perception of everyday objects. An additional aim of the experiment was to identify, if possible, some of the factors that influence the perception of the illusion in the real-world. Still another aim of the study was to see how well psychophysical experiments of this type can be done under real-world conditions.

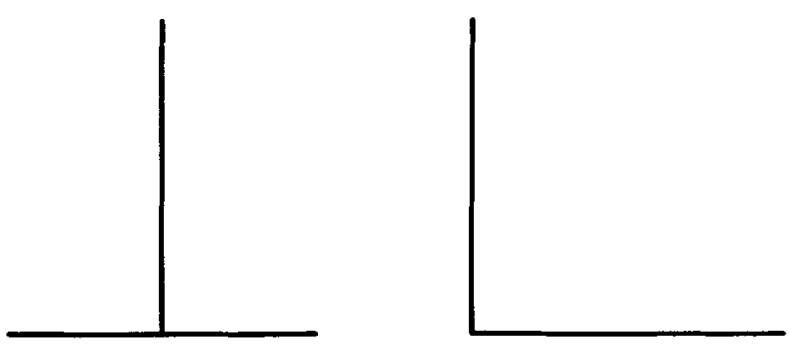

Fig. 1. Two conventional forms of the vertical-horizontal illusion. The vertical lines are usually judged to be longer than the horizontal lines although all are of equal length. 

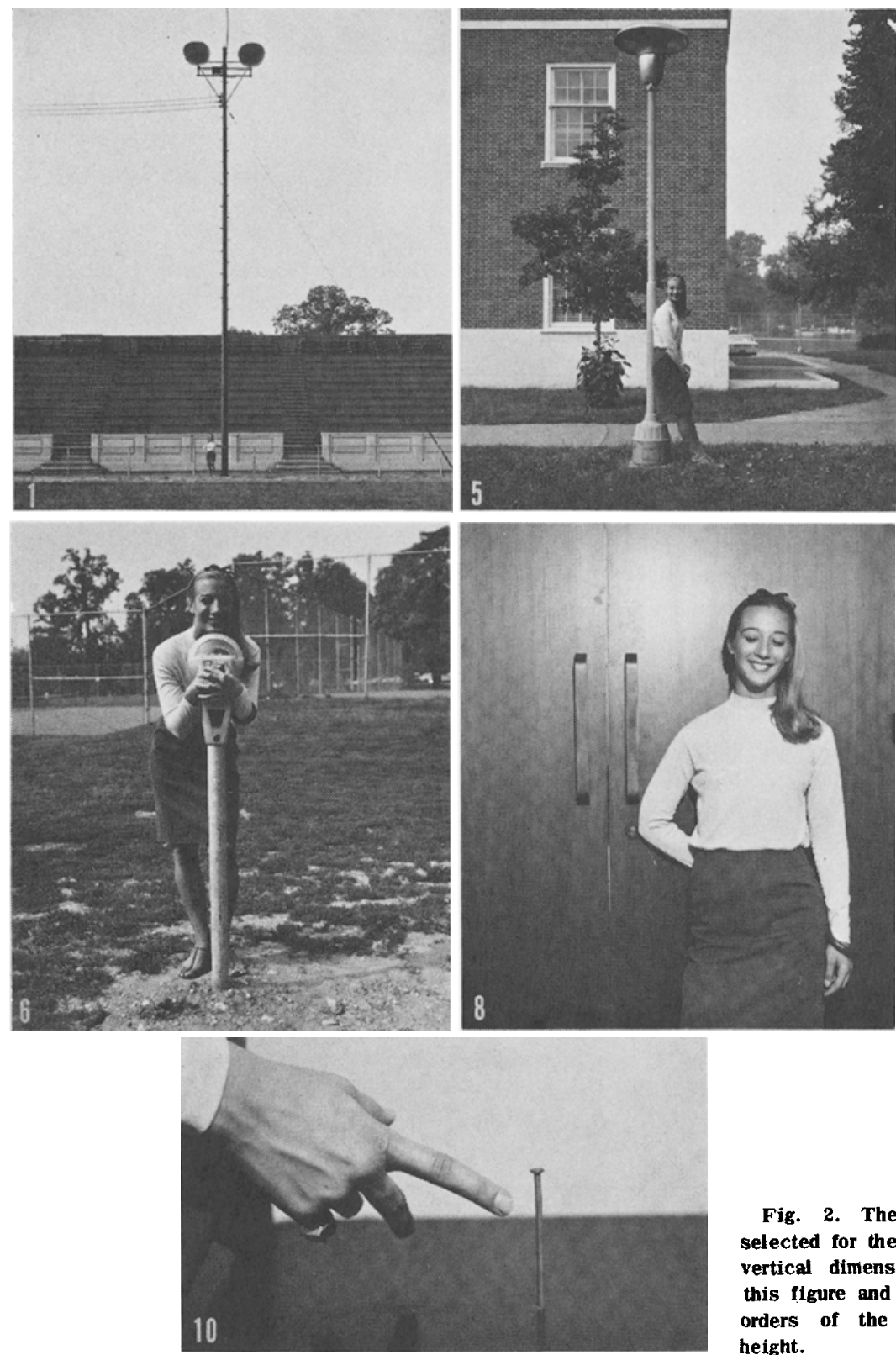

Fig. 2. These five objects were selected for their predominantly linear, vertical dimensions. The numerals in this figure and in Fig. 3 are the rank orders of the objects according to height.

\section{Subjects}

\section{METHOD}

The Ss were 20 volunteers, 10 male and 10 female, recruited by local advertising from among the students attending summer school at The Johns Hopkins University. Except for a 31-year old male, the Ss were between the ages of 18 and 26. Each S participated in a 1- to 1-1/2 hr. session on each of three consecutive days. All were paid for their participation in the study.

\section{Stimuli}

Ten stimulus objects, ranging from a few inches to over $60 \mathrm{ft}$. in height, were selected on the campus of the university. The objects were chosen to form two groups. The first group (see Fig. 2) was selected for the linear, vertical dimensions that have characterized the stimulus objects commonly used in laboratory experiments. These objects and their approximate heights were: a football light standard (61 ft.), a lamp post (13 ft.), a parking meter (4.5 ft.), a door handle (16 in.), and a nail in a table (3 in.). The objects in the second group (see Fig. 3) were much more complex in form or were not as easily differentiated from their backgrounds as the objects in the first group. They included the height of a classroom building from the base to the chimney (58 $\mathrm{ft}$.), a large tree ( $52 \mathrm{ft}$.), an ornamental brick column (19 ft.), a fire hydrant (27 in.), and the vertical edge of a step (5 in.). The 

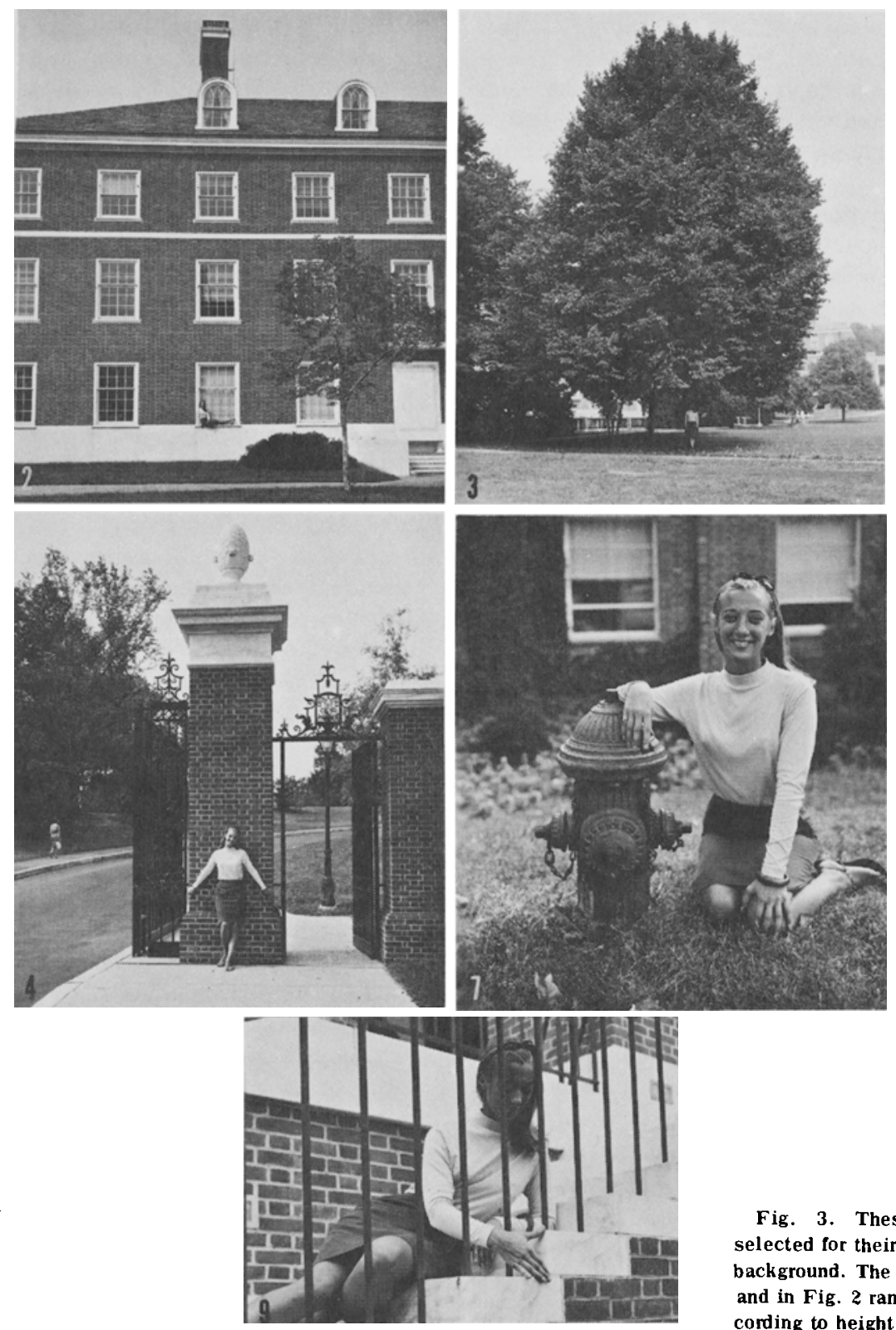

Fig. 3. These five objects were selected for their complexity of form and background. The numerals in this figure and in Fig. 2 rank order the objects according to height.

objects in one group were roughly matched in height to the objects in the other group.

\section{Procedure}

The objects were viewed from a standard viewing distance, a distance at which each object subtended a visual angle of about $22^{\circ}$. The $\mathrm{S}$ was asked to judge the height of each object by indicating where the top of it would be if it were tipped over on its side. He was instructed to make his estimate without the use of any physical aids such as fingers or sticks. The psychophysical procedure was a variation of the method of average error. The $\mathrm{E}$ started at the base of the object with a marker. The $\mathrm{S}$ directed the $\mathrm{E}$ to move out from the object at a right angle to the S's line of vision. The directions were given by voice if the objects were nearby, or by arm movements if they were distant. The $\mathrm{E}$ moved away from the object until the $\mathrm{S}$ was satisfied that the horizontal distance marked off by the $E$ was equal to the vertical height of the object being viewed. The $S$ had unlimited time to make his judgments and was allowed to make as many adjustments in either direction as he felt were necessary to satisfy him. This procedure was followed for all 10 objects each day and was repeated on three consecutive days. The Ss were run one at a time and the testing order for each day was counterbalanced in a 10 by 10 Latin square for the 10 male 
and the 10 female Ss. A different Latin square was used for each day.

At the conclusion of the third day of testing each $S$ was asked which objects he felt were the most difficult to judge and why. He was also asked to describe what procedures (if any) he used in making his estimates.

A word of explanation is in order concerning our psychophysical method. As mentioned earlier, the $\mathrm{E}$ always started at the base of the object and moved out from it. If we had used the textbook model for the method of average error we would have varied the starting position of the $E$ on alternate trials and used many such trials. However, there were several considerations that led us to use the method we did. The first is a practical one. The experiment had to be completed within a few weeks and we could not count on having the Ss for more than a few days. Moreover, to run through all 10 objects on one day took about an hour and a half and required a fair amount of walking from one place to another. Thus we felt that we could not take more than one observation per day per object.

A second consideration is that we were afraid that if we were to take many observations per object, a $S$ would establish anchor points for his estimates and remember them from estimation to estimation. A visually-rich environment such as we used is, of course, full of all sorts of possible reference points that could easily be remembered from day to day.

Given then that we could use only a very few observations per object, it is likely that the E himself might establish an anchor point for the $S$, no matter where he started. Under these circumstances it seemed to us that we could best reduce bias from this source by having the $\mathrm{E}$ always start at the base of the object. Finally, because of space limitations, it was not possible in some cases to start far enough away from the object to satisfy the ideal textbook requirements for this psychophysical method.

Table 1. Mean percentage errors of estimation averaged across all objects and all days for the $20 \mathrm{Ss}$. The standard deviations are the averages of 10 standard deviations, each of which is based on the three estimations made for each object on the three different days.

\begin{tabular}{lrrr}
\multicolumn{2}{c}{$\begin{array}{c}\text { Male } \\
\text { Subjects }\end{array}$} & \multicolumn{2}{c}{$\begin{array}{c}\text { Female } \\
\text { Subjects }\end{array}$} \\
\cline { 2 - 4 } & S.D. & Mean & S.D. \\
\hline 18.54 & 9.7 & 53.69 & 21.2 \\
12.71 & 10.6 & 31.38 & 22.8 \\
12.04 & 7.4 & 18.83 & 14.7 \\
6.94 & 16.8 & 10.95 & 12.7 \\
0.85 & 6.8 & 7.50 & 7.0 \\
-0.18 & 8.0 & 5.72 & 5.3 \\
-0.53 & 9.3 & 3.96 & 7.7 \\
-1.80 & 12.0 & 2.31 & 10.9 \\
-2.44 & 15.2 & 0.72 & 9.9 \\
-11.43 & 9.7 & -5.27 & 13.8 \\
Overall Mean $=8.22(p<0.05)$ & & \\
\hline
\end{tabular}

We are fully aware of the consequences of using the method of average error with trials in an increasing direction only. It is possible that our results are influenced by our method. However, it must be kept in mind that Ss could, and did, make adjustments in either direction. This procedure should have compensated to some extent for any directional influences.

\section{Treatment of the data}

Each $S$ made one observation per day on each of the 10 objects. These observations were repeated for three consecutive days making a total of 30 observations per S. Since we used $20 \mathrm{Ss}$ our raw data consist of 600 measurements. Each measurement was the horizontal distance from the base of the object to where the $S$ estimated the top of the object would be if it were tipped on its side. The true height of each object was then subtracted from its estimated height to give an error of estimation for each object. A negative error indicates an underestimation and a positive error indicates an overestimation. The error was then converted into a percentage of the true height of the object. Whenever we refer to errors of estimation or, more simply, errors, later in this paper we mean the percentage errors calculated as described above.

\section{RESULTS}

Using these percentage errors of estimation, arithmetic means were computed for the 30 estimations (10 on each of three days) made by each $\mathrm{S}$. These means are given in Table 1. A standard deviation was computed for the estimations on each object made on each of the three days. The 10 standard deviations for each object were then averaged to give the variability of each S's estimates independent of the variability between objects. These data are also given in Table 1. A t-test made on the overall mean shows that it is significantly different from zero, that is, on the average Ss significantly overestimated the vertical distances $(t=2.52, p<.05)$.

Mean errors were also calculated for each object using the data for all days and all Ss. These means are shown in Fig. 4. The mean error of estimation for the football light standard, the bullding, the brick column, the lamp post, and the parking meter were significantly positive (ps< .02, .01, .01, .001, and .001, respectively). Errors for the door handle and the edge of the step were significantly negative (ps $<.01$ and .001 , respectively) showing the reverse of the illusion. The errors for the tree, hydrant, and nail were not significantly different from zero.

An analysis of variance of the percentage errors of estimation (Table 2) shows that the differences among the objects are highly significant $(p<.001)$. As we remarked earlier, in selecting our stimulus objects we deliberately picked them to vary in height and in complexity of contour. Since these variables were de- 
Table 2. Analysis of variance of percentage errors of estimation

\begin{tabular}{|c|c|c|c|c|}
\hline Souree & es of Variotion & $\begin{array}{l}\text { Degrees of } \\
\text { freedom }\end{array}$ & MS & $F$ ratios \\
\hline I. & $\begin{array}{l}\text { Between subjects (Su) } \\
\text { A. Between sexes ( } \mathrm{Se}) \\
\text { B. Between subjeets of the same sex ( } \mathrm{Su}_{\text {we }} \text { ) }\end{array}$ & $\begin{array}{r}1 \\
1 \\
18\end{array}$ & $\begin{array}{r}6,263.35 \\
13,562.50 \\
5,857.84\end{array}$ & 2.32 \\
\hline II. & Between days (D) & 2 & 749.02 & 2.08 \\
\hline III. & $\begin{array}{l}\text { Between objects }(\mathrm{O}) \\
\text { A. Large vs. small objects }(\mathrm{Si}) \\
\text { B. Simple vs. complex contours (C) } \\
\text { C. All other orthogonal comparisons (R) }\end{array}$ & $\begin{array}{l}1 \\
1 \\
7\end{array}$ & $\begin{array}{r}7,625.65 \\
22,969.49 \\
1,186.70 \\
6,353.53\end{array}$ & $\begin{array}{l}16.33^{*} \\
49.20^{*} \\
2.54\end{array}$ \\
\hline IV. & 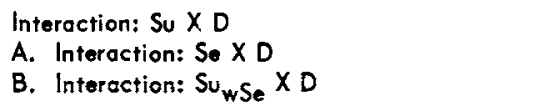 & $\begin{array}{r}38 \\
2 \\
36\end{array}$ & $\begin{array}{l}359.78 \\
428.14 \\
355.98\end{array}$ & \\
\hline v. & $\begin{array}{l}\text { Interaction: Su } \times 0 \\
\text { A. Interaction: Se } \times 0 \\
\text { B. Interaction: } \mathrm{Su}_{w S e} \times 0\end{array}$ & $\begin{array}{r}171 \quad 9 \\
162\end{array}$ & $\begin{array}{l}466.90 \\
807.08 \\
448.00\end{array}$ & 1.80 \\
\hline VI. I & Interaction: $D \times 0$ & 18 & 60.40 & \\
\hline VII. & $\begin{array}{l}\text { Interaction: Su X D XO } \\
\text { A. Interaction: Se X D XO } \\
\text { B. Interaction: Su } U_{w S} \times D \times O\end{array}$ & $\begin{array}{r}342 \\
18 \\
324\end{array}$ & $\begin{array}{l}73.23 \\
89.13 \\
72.34\end{array}$ & \\
\hline VIII. & Total & 599 & & \\
\hline
\end{tabular}

signed into the experiment, we have compared two orthogonal combinations, each with one degree of freedom, as part of the variance attributable to differences among the objects. One of these comparisons, item III.A. in Table 2, is between the five largest and five smallest objects. The second comparison, item III.B. in Table 2, is between objects with relatively simple contours (Objects 1, 5, 8, and 10) and those with complex contours (Objects 2, 4, 7, and 9). Note also that the objects with simple contours are each matched approximately in height to the objects with complex contours. Since no other comparisons were planned

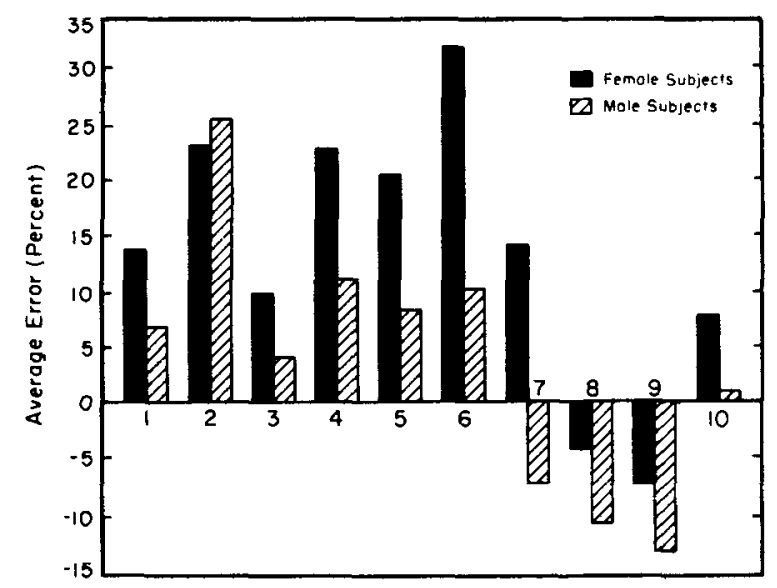

Fig. 4. Mean errors of estimation for each object. Each bar is the mean of 30 measurements: 10 Ss times three days. The numerals here correspond to those in Figs. 2 and 3. in this experiment, the remaining orthogonal comparisons, with 7 degrees of freedom, are pooled in Table 2 (item III.C.). The results of these additional comparisons show that there is a highly significant difference between the large and small objects, but that there is no significant difference between the simple and complex objects. The former finding is exactly the reverse of what Pan (1934) reported. Of course, it is important to note that Pan's results were obtained in the laboratory and with figures very much smaller than our objects.

Fraisse and Vautrey (1956) allowed unlimited time for observation, as we did, and found that females showed significantly greater overestimations than did the males. We were not able to confirm this finding in our data. Note, finally, that Table 2 shows no significant variation between days (and so by implication, no significant practice effect) and no significant interactions whatsoever.

\section{DISCUSSION}

A primary objective of this experiment was to determine whether the vertical-horizontal illusion can be demonstrated in a visually-rich environment. The best answer seems to be an equivocal "Yes." That is, it appears that the illusion depends largely upon the peculiar characteristics of the objects themselves and on factors that may be present in the backgrounds of the objects. For some objects the illusion appeared to be very strong while for others it either did not appear at all or was even reversed. The large amount 
Table 3. Variances and standard deviations of the percentage errors of estimation averaged across Ss

\begin{tabular}{lcc} 
Object & Variance & $\begin{array}{r}\text { Standard } \\
\text { deviation }\end{array}$ \\
\hline Light standard & 252.4 & 13.1 \\
Building & 181.3 & 11.8 \\
Tree & 307.3 & 13.8 \\
Brick column & 263.1 & 14.2 \\
Lamp post & 231.1 & 12.8 \\
Parking meter & 336.2 & 14.3 \\
Hydrant & 224.1 & 12.4 \\
Door handle & 100.5 & 8.3 \\
Step & 72.4 & 6.8 \\
Nail & 96.4 & 8.4 \\
\hline
\end{tabular}

of variation in the mean estimates among the objects indicates that the illusion depends very much on influences that have never been considered before or, at most, oniy vaguely alluded to in the experimental literature. We have tried to identify some of these influences. Before describing them, however, it is important to point out that, because of the nature of our experiment, they are, at present, only conjectures. Further research is required to determine whether we have, indeed, Identified significant influences on the illusion.

One factor that appears to be of some importance is the angle of regard, 1.e., the angle between the S's line of sight to the object and the horizontal. For the large, distant objects the line of sight was essentially straight ahead. On the other hand, for the five smaller objects, the $\mathrm{Sg}$ had to look either up or down at some angle to the object. For example, although the step was at eye level for some Ss, It was above eye level for others. As another example, the nail was stuck into a table and the $S$ was seated about 8 in. away. The S's eyes were about a foot or so higher than the top of the nail forcing him to look down with a considerable foreshortening of the retinal image. This was also the case for the parking meter, fire hydrant, and door handle although the foreshortening was not as pronounced for these three objects. If the angle of regard were the only factor interacting with the illusory effect, estimations for the nail should have been affected more than estimations for the hydrant because the foreshortening of the retinal image of the former was greater than that of the latter. The data (see Fig. 4) do not show this to be the case. Note, further, that the illusion was apparent for the parking meter although it also fell below eye level for all Ss. Such inconsistencies suggest that there were some other factors, possibly size constancy, influencing the estimations of these objects.

Estimations of the height of the step suggest one of these other influences, 1.e., that of anchor points. We can best describe this influence with a description of the estimating procedure. The $E$ held a pencil alongside the edge of the step as a marker. The $S$ directed the $\mathrm{E}$ to move the pencil along the horizontal edge of the step to mark off the estimated height. At a few inches along the edge was a small chip in the marble. In several instances Ss referred to the chip when making their estimates. Because the distance from the chip to the edge of the step was approximately an inch less than the height of the step, the Ss' estimates may have been smaller than they otherwise might have been. This would also constrain the range and, as a result, the variances of the errors.

To determine whether the variances of the errors differed significantly, the three errors of estimation for each day were used to calculate a variance and a standard deviation for each S's estimates of each object. The variances and standard deviations for each object were then averaged for all Ss. These averages are given in Table 3. An analysis of variance using the variances, and another analysis using the standard deviations, yielded parallel results. They show that estimates for some objects had indeed been significantly constrained ( $p$ for the variances $<.05$, $p$ for the standard deviations $<.025)$.

Of the 10 objects the three smallest ones had the three lowest variances. The step, the second smallest object, had the lowest variance. The other two objects with low variances were the nall and the door handle. In the case of the latter two objects, however, Ss did not refer to anchor points nearly as often as they did for the step.

Table 4. Analysis of variance on the variances of the errors of estimation

\begin{tabular}{|c|c|c|c|}
\hline Sources of Variation & $\begin{array}{c}\text { Degrees of } \\
\text { freedom }\end{array}$ & MS & F ratios \\
\hline 1. Subjects (Su) & 19 & & \\
\hline A. Between sexes (Se) & 1 & $476,239.20$ & 1.60 \\
\hline B. Between subjects within sexes $\left(\mathrm{Su}_{w} \mathrm{Se}_{\mathrm{e}}\right)$ & 18 & $297,421.93$ & \\
\hline II. Between objects $(0)$ & 9 & $167,100.33$ & $2.20 *$ \\
\hline III. Interaction: Subjects $\times$ Objects (Su $\times 0$ ) & 171 & & \\
\hline $\begin{array}{l}\text { A. Interoction: Se } \times 0 \\
\text { B. Interoction: } S_{w S e} \times 0\end{array}$ & $\begin{array}{r}9 \\
162\end{array}$ & $\begin{array}{l}37,412.32 \\
76,030.50\end{array}$ & $<1$ \\
\hline IV. Total & 199 & & \\
\hline
\end{tabular}


The three objects with the lowest variances (nail In a table, door handle on a door, edge of a step) were similar in one respect-they had a relatively uniform background as compared with the backgrounds for the other objects. For this reason these three objects almost seem to be a compromise between the usual laboratory stimuli with their uniform backgrounds and the other objects with their unusually rich environments. The plain background provides no opportunity at all for anchoring, while the rich visual environments of the other objects provide abundant opportunity for anchoring-but unless there happens to be an especially prominent point, anchoring is either unlikely or else does not always occur on the same points for all Ss. A reasonable speculation might be that a stray mark, chip, or scratch will more likely serve as an anchor point if it is not imbedded in a large amount of visual noise originating from the background of the object.

Although we believe our speculations about anchor points to be correct, there may have been still other factors that influenced the estimations. Our analysis showed a significant effect due to size, i.e., estimates for the five largest objects showed a greater illusory effect than estimates for the five smaller ones. There is the possibility, therefore, that size of object per se may have been partly responsible for the lower variances. At this point, however, we cannot untangle the effects of these factors from our data and our conjectures must remain just that. In any case, we believe that the presence of an anchor point in the case of the step did indeed have a marked effect on the estimations made for that object.

There was one other object, the tree, for which estimations did not differ significantly from zero. None of the considerations we have discussed so far seems adequate to account for this result. It appears that in this case again there must have been other influences at work. One possibility might be the particular shape of the tree itself, although precisely what there is about the shape of the tree that produced this outcome is hard to say. Recall, however, that we did not find a difference between our simple and complex objects. Perhaps a more careful definition of complexity would yield significant results.

It is often the case in experiments on illusions that individual differences are large (see, for example, Finger \& Spelt, 1947; and Pollock \& Chapanis, 1952). This experiment is no exception (see Table 1). We tried to isolate some of the possible sources for these large variations among $S s$ by asking them at the conclusion of their third day of testing what methods, if any, they had used for their estimates. About $25 \%$ of the Ss used an imaginary arc of fall. About $50 \%$ of the Ss used the height of the object and the estimated horizontal distance to form an imaginary isosceles triangle. The remaining Ss did not use any particular method at all, or else used a method that did not fall into either of the other two categories. Mean estimates for these three groups did not differ significantly.
In short, it does not appear that the large individual differences in the lllusion can be explained by the various methods used by the Ss to make their estimations.

A secondary purpose of the experiment was to find our how well psychophysical experiments of this type can be done under real-world conditions. The present experiment demonstrated that a variation of the method of average error could reasonably be used in experiments conducted outside of the laboratory environment. Doing experiments under real-world conditions is, however, much more difficult than doing comparable studies in the laboratory. It is more difficult to control or to anticipate all the factors that may influence the results of a real-world experiment. This weakness of such experimentation is, however, one of its major strengths. By doing this experiment with real objects we feel that we have been able to make statements of much greater generality than would have resulted from a laboratory study. At the same time, the use of real-world objects has given us insights into important factors that may influence this illusion. These insights would not have been forthcoming from the typical laboratory experiment because the factors eliciting them would ordinarily have been controlled out of existence and so would never have been able to exert their influence.

In conclusion it seems that the vertical-horizontal illusion can be demonstrated under certain conditions present in the real world. This paper also suggests some of the factors that may influence it, although these factors alone do not appear sufficient to explain specific results. The role of these factorssize, size constancy, angle of regard, anchor pointsand the role of other, as yet, unknown factors may eventually be better defined by future research.

\section{References}

Finger, F. W., spelt, D. K. The illustration of the horizontalvertical illusion. J. exp. Psychol., 1947, 37, 243-250.

Fraisse, P., \& Vautrey, P. The influence of age, sex, and specialized training on the vertical-horizontal illusion. Quart. J. exp. Psychol., 1956, 8, 114-120.

Künnapas, T. M. An analysis of the "vertical-horizontal illusion." J. exp. Psychol., 1955, 49, 134-140.

Kunnapas, T. M. The vertical-horizontal illusion and the visual field. J. exp. Psychol., 1957, 53, 405-407.

Pan, S. The vertical-horizontal illusion. N.C.J. Psychol, nat, cent. Univ., 1934, 1, 125-128.

Pollock, W. T., \& Chapanis, A. The apparent length of a line as a function of its inclination. Quart. J. exp. Psychol., 1952, 4, 170-178.

Sleight, R. B., \& Austin, T. R. The horizontal-vertical illusion in plane geometric figures. J. Psychol., 1952, 33, 279-287.

\section{Note}

1. The work reported in this article was done under Contract Nonr4010(03) between the Office of Naval Research and The Johns Hopkins University. This is Report No. 17 under that eontract. Reproduction in whole or in part is permitted for any purposes of the United States Government.

(Accepted for publication March 3, 1967.) 